\title{
Complete genome sequence of Haliscomenobacter hydrossis type strain $\left(\mathrm{O}^{\top}\right)$
}

\author{
Hajnalka Daligault ${ }^{1,2}$, Alla Lapidus ${ }^{1}$, Ahmet Zeytun ${ }^{1,2}$, Matt Nolan ${ }^{1}$, Susan Lucas ${ }^{1}$, Tijana \\ Glavina Del Rio ${ }^{1}$, Hope Tice ${ }^{1}$, Jan-Fang Cheng ${ }^{1}$, Roxanne Tapia ${ }^{1,2}$, Cliff Han ${ }^{1,2}$, Lynne \\ Goodwin ${ }^{1,2}$, Sam Pitluck ${ }^{1}$, Konstantinos Liolios ${ }^{1}$, Ioanna Pagani ${ }^{1}$, Natalia Ivanova ${ }^{1}$, Marcel \\ Huntemann ${ }^{1}$, Konstantinos Mavromatis ${ }^{1}$, Natalia Mikhailova ${ }^{1}$, Amrita Pati ${ }^{1}$, Amy Chen ${ }^{3}$, \\ Krishna Palaniappan ${ }^{3}$, Miriam Land ${ }^{1,4}$, Loren Hauser ${ }^{1,4}$, Evelyne-Marie Brambilla ${ }^{5}$, Manfred \\ Rohde $^{6}$, Susanne Verbarg ${ }^{5}$, Markus Göker ${ }^{5}$, James Bristow ${ }^{1}$, Jonathan A. Eisen ${ }^{1,7}$, Victor \\ Markowitz ${ }^{4}$, Philip Hugenholtz ${ }^{1,8}$, Nikos C. Kyrpides ${ }^{1}$, Hans-Peter Klenk ${ }^{5 *}$, and Tanja \\ Woyke $^{1}$ \\ ${ }^{1}$ DOE Joint Genome Institute, Walnut Creek, California, USA \\ ${ }^{2}$ Los Alamos National Laboratory, Bioscience Division, Los Alamos, New Mexico, USA \\ ${ }^{3}$ Biological Data Management and Technology Center, Lawrence Berkeley National \\ Laboratory, Berkeley, California, USA \\ ${ }^{4}$ Oak Ridge National Laboratory, Oak Ridge, Tennessee, USA \\ ${ }^{5}$ DSMZ - German Collection of Microorganisms and Cell Cultures GmbH, Braunschweig, \\ Germany \\ ${ }^{6} \mathrm{HZI}$ - Helmholtz Centre for Infection Research, Braunschweig, Germany \\ ${ }^{7}$ University of California Davis Genome Center, Davis, California, USA \\ ${ }^{8}$ Australian Centre for Ecogenomics, School of Chemistry and Molecular Biosciences, The \\ University of Queensland, Brisbane, Australia \\ *Corresponding author: Hans-Peter Klenk
}

Keywords: strictly aerobic, non-motile, Gram-negative, branching, sheathed, mesophilic, chemoorganotrophic, "Saprospiraceae", GEBA

\begin{abstract}
Haliscomenobacter hydrossis van Veen et al. 1973 is the type species of the genus Haliscomenobacter, which belongs to order "Sphingobacteriales". The species is of interest because of its isolated phylogenetic location in the tree of life, especially the so far genomically uncharted part of it, and because the organism grows in a thin, hardly visible hyaline sheath. Members of the species were isolated from fresh water of lakes and from ditch water. The genome of $H$. hydrossis is the first completed genome sequence reported from a member of the family "Saprospiraceae". The 8,771,651 bp long genome with its three plasmids of $92 \mathrm{kbp}$, $144 \mathrm{kbp}$ and $164 \mathrm{kbp}$ length contains 6,848 protein-coding and 60 RNA genes, and is a part of the Genomic Encyclopedia of Bacteria and Archaea project.
\end{abstract}

\section{Introduction}

Strain $0^{\mathrm{T}}(=$ DSM $1100=$ ATCC 27775 ) is the type strain of Haliscomenobacter hydrossis which is the type and only species within the genus Haliscomenobacter $[1,2]$. The generic name derives from the Greek word haliskomai, to be imprisoned, and the Neo-Latin bacter, a rod, meaning the imprisoned rod. The species epithet is derived from the Greek word hudôr, water, and Oss, a town in the Netherlands, hydrossis, from water of Oss. The imprisoned rod from the water of Oss. Five morphologically and physiologically congruent strains belonging to the species, including the type strain $\mathrm{O}^{\mathrm{T}}$, were isolated from activated sludge samples in the early 1970s [1]. H. hydrossis was sporadically ob- served in aeration tanks of sewage treatment plants in Germany [3] and in paper industry wastewater treatment plants in France [4]. As a recent biotechnological application, biomass bulking caused by $H$. hydrossis was controlled by lytic bacteriophages [5]. An improved high quality draft sequence of Saprospira grandis strain Sa g1 (=HR1, DSM 2844, GOLD ID Gi033955) is the only other genomic information currently available from the family "Saprospiraceae". Here we present a summary classification and a set of features for $H$. hydrossis $\mathrm{O}^{\mathrm{T}}$, together with the description of the complete genomic sequencing and annotation. 


\section{Classification and features}

The single genomic $16 \mathrm{~S}$ rRNA sequence of $H$. hydrossis $\mathrm{O}^{\mathrm{T}}$ was compared using NCBI BLAST [6] under default settings (e.g., considering only the highscoring segment pairs (HSPs) from the best 250 hits) with the most recent release of the Greengenes database [7] and the relative frequencies of taxa and keywords (reduced to their stem [8]) were determined, weighted by BLAST scores. The most frequently occurring genera were Haliscomenobacter (83.9\%) and Lewinella (16.1\%) (3 hits in total). Regarding the two hits to sequences from members of the species, the average identity within HSPs was 99.2\%, whereas the average coverage by HSPs was $98.1 \%$. Among all other species, the one yielding the highest score was Lewinella antarctica (EF554367), which corresponded to an identity of $89.1 \%$ and an HSP coverage of $66.6 \%$. (Note that the Greengenes database uses the INSDC (= EMBL/NCBI/DDBJ) annotation, which is not an authoritative source for nomenclature or classification). The highest-scoring environmental sequence was AJ786323 ('Lake Wolfgangsee freshwater enrichment clone MSWolf2- $\mathrm{H}^{\prime}$ ), which showed an identity of $98.8 \%$ and an HSP coverage of $97.9 \%$. The most frequently occurring keywords within the labels of environmental samples which yielded hits were 'lake' (10.6\%), 'tin' (5.3\%), 'microbi' (3.4\%), 'freshwat' (3.2\%) and 'mat' (3.2\%) (247 hits in total). The most frequently occurring keywords within the labels of environmental samples which yielded hits of a higher score than the highest scoring species were 'lake' (11.1\%), 'tin' (5.6\%), 'microbi' (3.5\%), 'freshwat' (3.4\%) and 'mat' (3.3\%) (225 hits in total). These keywords reflect the ecological properties reported for strain $\mathrm{O}^{\mathrm{T}}$ in the original description [1].

Figure 1 shows the phylogenetic neighborhood of $H$. hydrossis in a $16 \mathrm{~S}$ rRNA based tree. The sequences of the two 16S rRNA gene copies in the genome differ from each other by two nucleotides and do not differ from the previously published $16 \mathrm{~S}$ rRNA sequence AJ784892, which contains two ambiguous base calls.

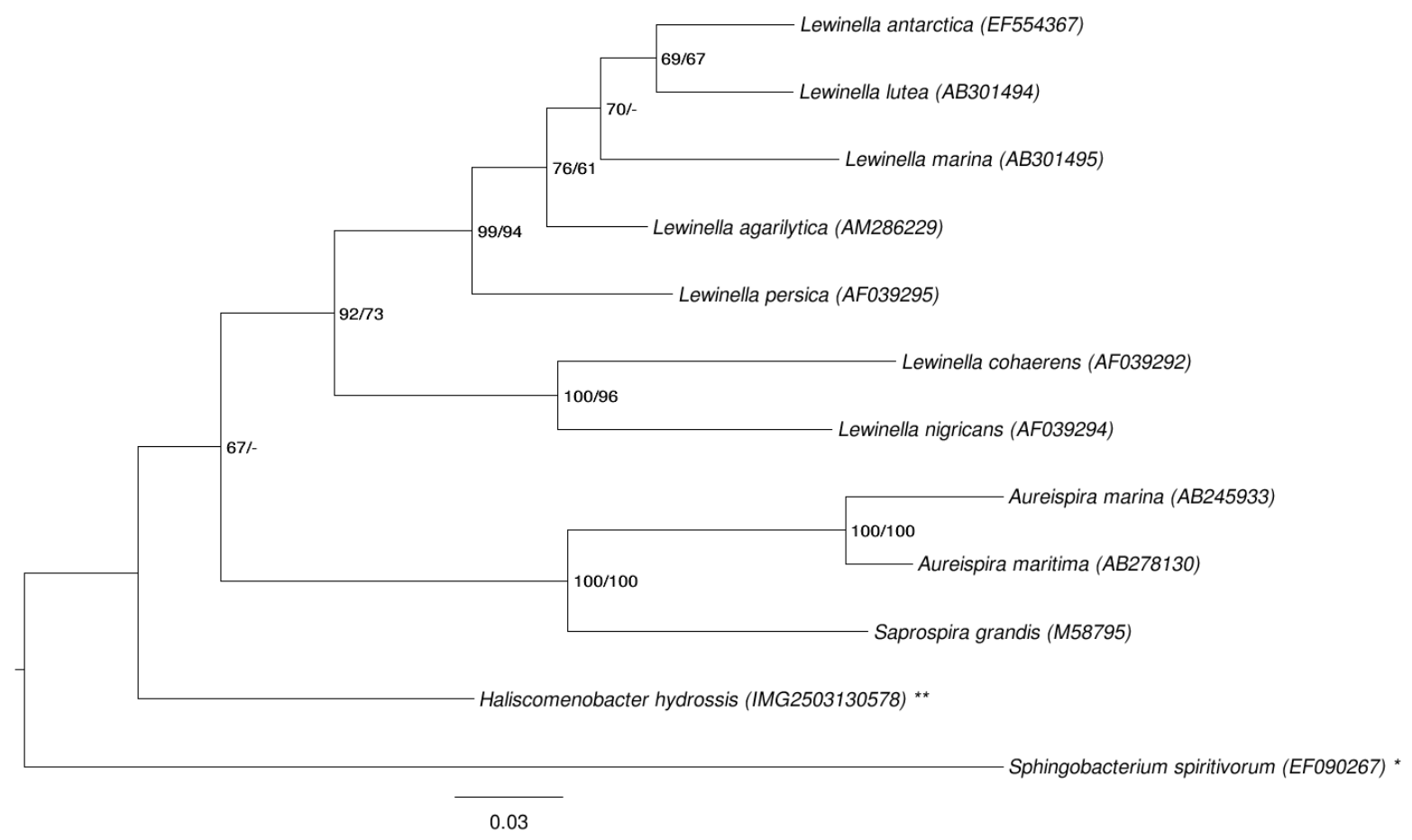

Figure 1. Phylogenetic tree highlighting the position of $H$. hydrossis relative to the type strains of the other species within the family "Saprospiraceae". The tree was inferred from 1,399 aligned characters $[9,10]$ of the $16 \mathrm{~S}$ rRNA gene sequence under the maximum likelihood (ML) criterion [11]. Rooting was done initially using the midpoint method [12] and then checked for its agreement with the current classification (Table 1). The branches are scaled in terms of the expected number of substitutions per site. Numbers adjacent to the branches are support values from 150 maximum likelihood bootstrap replicates [13] (left) and from 1,000 maximum parsimony bootstrap replicates [14] (right) if larger than 60\%. Lineages with type strain genome sequencing projects registered in GOLD [15] are labeled with one asterisk, those also listed as 'Complete and Published' with two asterisks. 
The cells of $H$. hydrossis are rod-shaped, 0.35 $0.45 \mu \mathrm{m}$ wide and 3 - $5 \mu \mathrm{l}$ long, mostly occurring in chains and nearly always enclosed by a narrow hyaline sheath (Figure 2) [1]. The sheath is sometimes disrupted by branching cells [1]. Flagella were never visible in EM images nor was motility ever observed [1]. Growing bacteria excrete so far unidentified polysaccharides [1]. Strain $\mathrm{O}^{\mathrm{T}}$ grows strictly aerobically and produces intracellular carotenoid pigments [1]. Optimal growth temperature was $26^{\circ} \mathrm{C}$, with a span of $8-30^{\circ} \mathrm{C}$ [1]. Optimal $\mathrm{pH}$ for growth was 7.5 [1]. Organic acids, peptides, proteins, mono- and polysaccharides were reported as carbon and energy sources [1]. Starch and gelatine were decomposed by all strains of the species [1], sorbitol, glycerol, lactate, acetate, succinate and $\beta$-hydroxybutyrate were not utilized [1]. The authors of the original description of the strain suggested that $\mathrm{O}^{\mathrm{T}}$ accumulates polysaccharides either intra- or extracellularily [1].

\section{Chemotaxonomy}

Nothing is known about the structure of the cell wall of $H$. hydrossis. The six major fatty acids of strain $\mathrm{O}^{\mathrm{T}}$ were iso- $\mathrm{C}_{15: 0}$ 3-0 $(22.8 \%)$, iso- $\mathrm{C}_{15: 0}$ (21.0\%), $\mathrm{C}_{16: 1}(17.3 \%)$, iso- $\mathrm{C}_{15: 0}$ 2-он $(15.5 \%)$, and $\mathrm{C}_{18: 0}(6.9 \%)$ and $\mathrm{C}_{16: 0}(5.7 \%)$ [24]. The type strain contained significantly more hydroxylated fatty acids than several analyzed reference strains from the genus [24]. Observed quinones were mainly of the MK-7 type (70-90\%), with 10-30\% MK-6 [24].

\section{Genome sequencing and annotation Genome project history}

This organism was selected for sequencing on the basis of its phylogenetic position [25], and is part of the Genomic Encyclopedia of Bacteria and Archaea project [26]. The genome project is deposited in the Genome On Line Database [15] and the complete genome sequence is deposited in GenBank. Sequencing, finishing and annotation were performed by the DOE Joint Genome Institute (JGI). A summary of the project information is shown in Table 2.

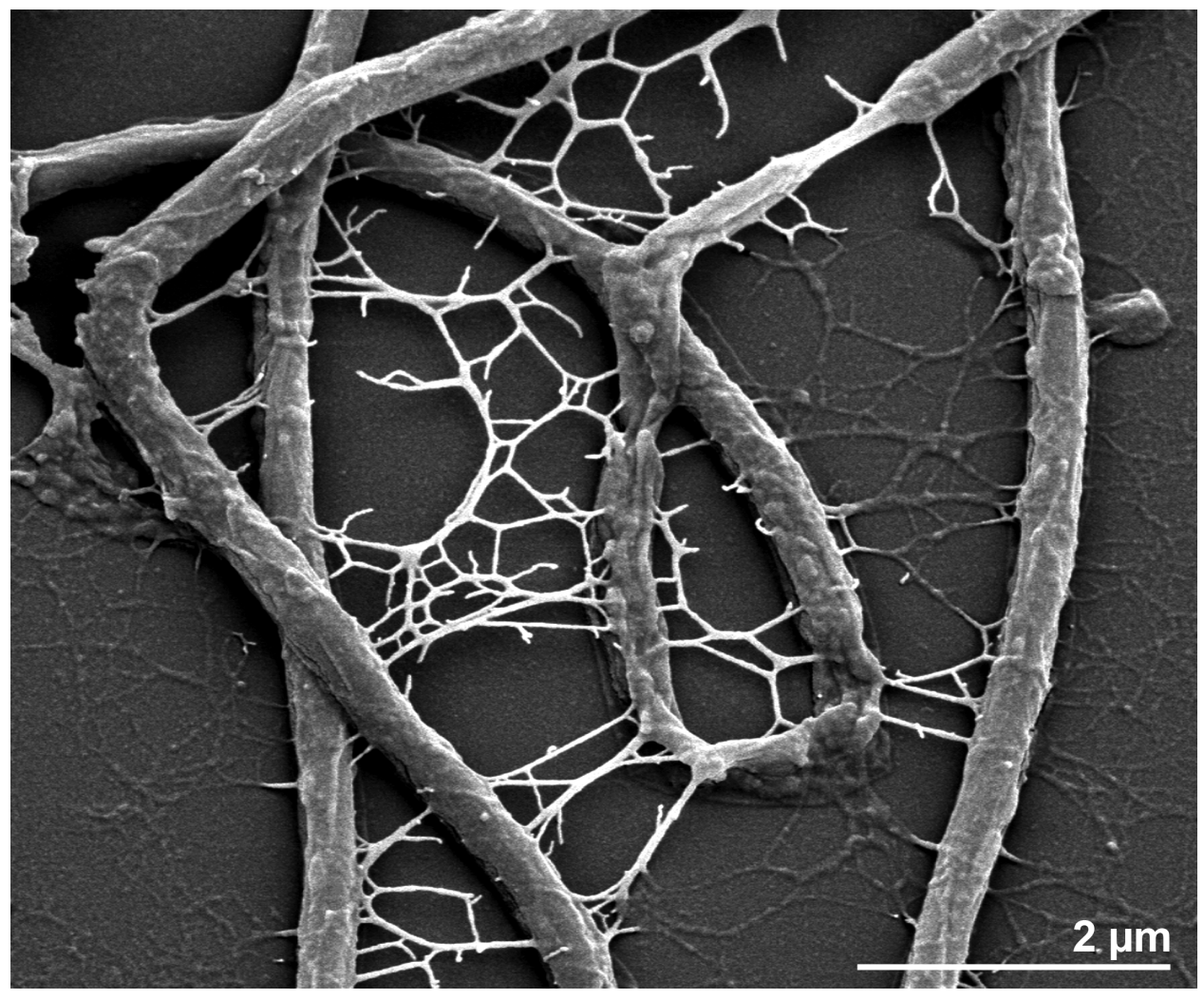

Figure 2. Scanning electron micrograph of $H$. hydrossis $\mathrm{O}^{\top}$ 
Table 1. Classification and general features of $H$. hydrossis $\mathrm{O}^{\top}$ according to the MIGS recommendations [16] and the NamesforLife database [17].

\begin{tabular}{|c|c|c|c|}
\hline MIGS ID & Property & Term & Evidence code \\
\hline & & Domain Bacteria & TAS [18] \\
\hline & & Phylum Bacteroidetes & TAS [19] \\
\hline & & Class 'Sphingobacteria' & TAS [20] \\
\hline & & Order 'Spingobacteriales' & TAS [20] \\
\hline & & Family 'Saprospiraceae' & TAS [21] \\
\hline & & Genus Haliscomenobacter & TAS $[1,2]$ \\
\hline & & Species Haliscomenobacter hydrossis & TAS [1] \\
\hline & Current classification & Type strain $\mathrm{O}$ & TAS $[1,2]$ \\
\hline & Gram stain & negative & TAS [1] \\
\hline & Cell shape & rod-shaped with a hyaline sheath & TAS [1] \\
\hline & Motility & non-motile & TAS [1] \\
\hline & Sporulation & not reported & \\
\hline & Temperature range & $8-30^{\circ} \mathrm{C}$ & TAS [1] \\
\hline & Optimum temperature & $26^{\circ} \mathrm{C}$ & TAS [1] \\
\hline & Salinity & not reported & \\
\hline \multirow[t]{3}{*}{ MIGS-22 } & Oxygen requirement & $\begin{array}{l}\text { strictly aerobic } \\
\text { organic acids, peptides, proteins, }\end{array}$ & TAS [1] \\
\hline & Carbon source & mono- and polysaccharides & TAS [1] \\
\hline & Energy metabolism & chemoorganotroph & TAS [1] \\
\hline MIGS-6 & Habitat & fresh water of lakes, ditch water & TAS [1] \\
\hline MIGS-15 & Biotic relationship & free-living & TAS [1] \\
\hline \multirow[t]{3}{*}{ MIGS-14 } & Pathogenicity & none & NAS \\
\hline & Biosafety level & 1 & TAS [22] \\
\hline & Isolation & bulking activated sludge & TAS [1] \\
\hline MIGS-4 & Geographic location & Oss, The Netherlands & TAS [1] \\
\hline MIGS-5 & Sample collection time & before 1973 & TAS [1] \\
\hline MIGS-4.1 & Latitude & 51.77 & NAS \\
\hline MIGS-4.2 & Longitude & 5.53 & NAS \\
\hline MIGS-4.3 & Depth & 0 , surface & TAS [1] \\
\hline MIGS-4.4 & Altitude & about $8 \mathrm{~m}$ & NAS \\
\hline
\end{tabular}

Evidence codes - IDA: Inferred from Direct Assay (first time in publication); TAS: Traceable Author Statement (i.e., a direct report exists in the literature); NAS: Non-traceable Author Statement (i.e., not directly observed for the living, isolated sample, but based on a generally accepted property for the species, or anecdotal evidence). These evidence codes are from of the Gene Ontology project [23]. If the evidence code is IDA, the property was directly observed by one of the authors or an expert mentioned in the acknowledgements.

\section{Growth conditions and DNA isolation}

H. hydrossis $\mathrm{O}^{\mathrm{T}}$, DSM 1100, was grown in DSMZ medium 134 (Haliscomenobacter Medium) [27] at $26^{\circ} \mathrm{C}$. DNA was isolated from $0.5-1 \mathrm{~g}$ of cell paste using MasterPure Gram-positive DNA purification kit (Epicentre MGP04100) following the standard protocol as recommended by the manufacturer, with modification st/DL for cell lysis as described in Wu et al. [26]. DNA is available through the DNA Bank Network [28].

\section{Genome sequencing and assembly}

The genome was sequenced using a combination of Illumina and 454 sequencing platforms. All general aspects of library construction and sequencing can be found at the JGI website [29]. Pyrosequencing reads were assembled using the Newbler assembler (Roche). The initial Newbler assembly consisting of 153 contigs in three scaffolds was converted into a phrap [30] assembly by making fake reads from the consensus, to collect the read pairs in the 454 paired end library. 
Illumina GAii sequencing data $(1,273.3 \mathrm{Mb})$ was assembled with Velvet [31] and the consensus sequences were shredded into $1.5 \mathrm{~kb}$ overlapped fake reads and assembled together with the 454 data. The 454 draft assembly was based on 369.3 $\mathrm{Mb} 454$ draft data and all of the 454 paired end data. Newbler parameters are -consed -a 50 -l 350 -g -m -ml 20. The Phred/Phrap/Consed software package [30] was used for sequence assembly and quality assessment in the subsequent finishing process. After the shotgun stage, reads were assembled with parallel phrap (High Performance Software, LLC). Possible mis-assemblies were corrected with gapResolution [29], Dupfinisher [32], or sequencing cloned bridging PCR fragments with subcloning. Gaps between contigs were closed by editing in Consed, by PCR and by Bubble PCR primer walks (J.-F. Chang, unpublished). A total of 589 additional reactions were necessary to close gaps and to raise the quality of the finished sequence. Illumina reads were also used to correct potential base errors and increase consensus quality using a software Polisher developed at JGI [33]. The error rate of the completed genome sequence is less than 1 in 100,000 . Together, the combination of the Illumina and 454 sequencing platforms provided $203.8 \times$ coverage of the genome. The final assembly contained 1,005,536 pyrosequence and 35,370,321 Illumina reads.

Table 2. Genome sequencing project information

\begin{tabular}{lll}
\hline MIGS ID & Property & Term \\
\hline MIGS-31 & Finishing quality & Finished \\
MIGS-28 & Libraries used & Four genomic libraries: 454 pyrosequence standard library, 454 PE \\
MIGS-29 & Sequencing platforms & Illumina GAii, 454 GS FLX Titanium \\
MIGS-31.2 & Sequencing coverage & 165.3 x Illumina; 38.5 x pyrosequence \\
MIGS-30 & Assemblers & Newbler version 2.3, Velvet version 0.7.63, phrap version SPS - 4.24 \\
MIGS-32 & Gene calling method & Prodigal 1.4, GenePRIMP \\
& & CP002691 (chromosome) \\
& & CP002692 (plasmid pHALHY01) \\
& & CP002693 (plasmid pHALHY02) \\
& INSDC ID & CP002694 (plasmid pHALHY03) \\
& Genbank Date of Release & May 9, 2011 \\
& GOLD ID & GC01752 \\
& NCBI project ID & 48289 \\
& Database: IMG-GEBA & 2504756004 \\
& Source material identifier & DSM 1100 \\
& Project relevance & Tree of Life, GEBA \\
\hline
\end{tabular}

\section{Genome annotation}

Genes were identified using Prodigal [34] as part of the Oak Ridge National Laboratory genome annotation pipeline, followed by a round of manual curation using the JGI GenePRIMP pipeline [35]. The predicted CDSs were translated and used to search the National Center for Biotechnology Information (NCBI) non-redundant database, UniProt, TIGR-Fam, Pfam, PRIAM, KEGG, COG, and InterPro databases. Additional gene prediction analysis and functional annotation was performed within the Integrated Microbial Genomes - Expert Review (IMG-ER) platform [36].

\section{Genome properties}

The genome consists of an 8,371,686 bp long circular chromosome and three plasmids of 164,019 bp, 143,757 bp and 92,189 bp length, respectively, with a $\mathrm{G}+\mathrm{C}$ content of $47.1 \%$ (Table 3 and Figure $3)$. Of the 6,918 genes predicted, 6,858 were protein-coding genes, and 60 RNAs; 106 pseudogenes were also identified. The majority of the proteincoding genes (58.6\%) were assigned with a putative function while the remaining ones were annotated as hypothetical proteins. The distribution of genes into COGs functional categories is presented in Table 4. 


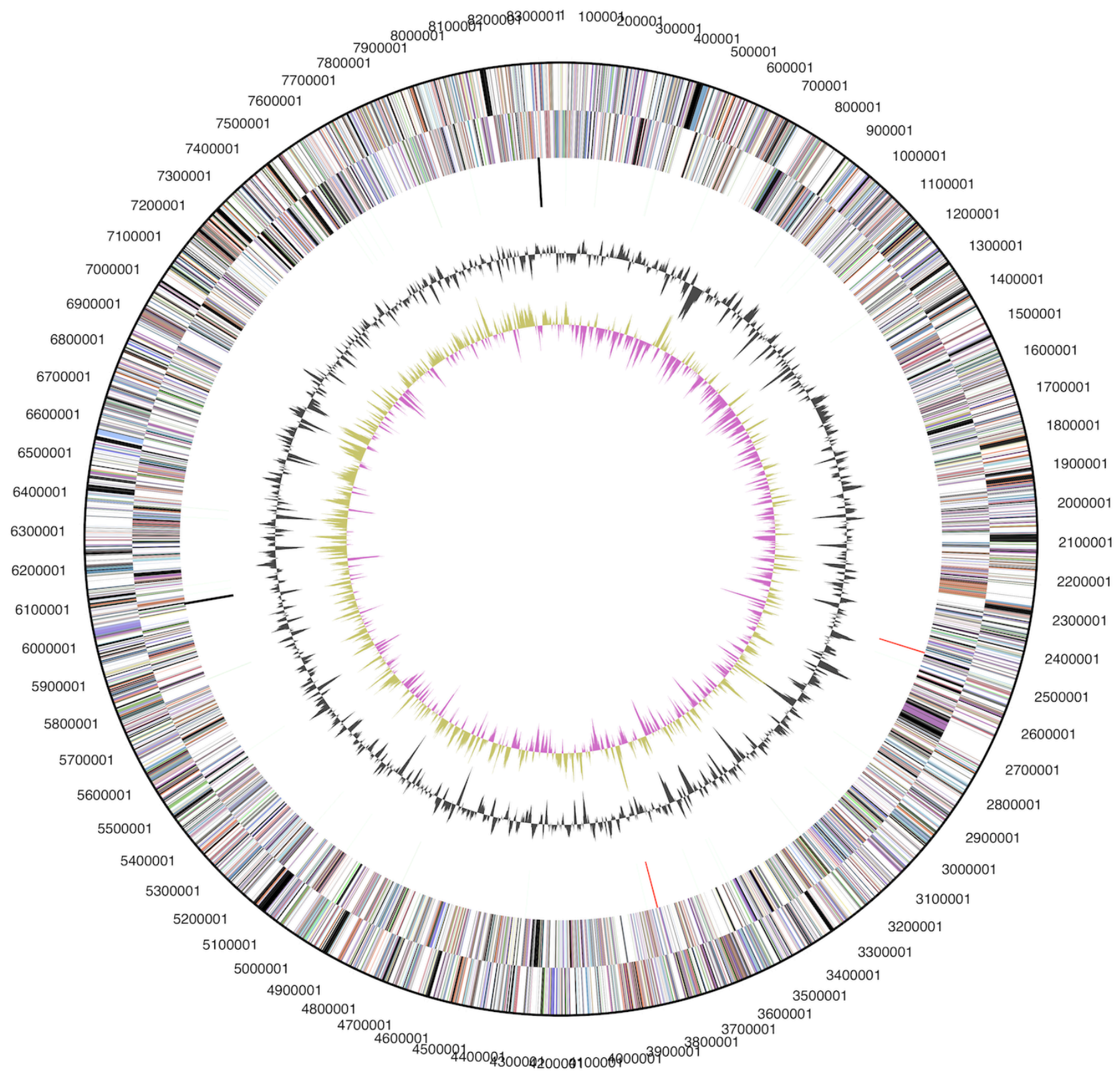

Figure 3. Graphical circular map of the chromosome (plasmid maps not shown). From outside to the center: Genes on forward strand (color by COG categories), Genes on reverse strand (color by COG categories), RNA genes (tRNAs green, rRNAs red, other RNAs black), GC content, GC skew. 
Table 3. Genome Statistics

\begin{tabular}{lrr}
\hline Attribute & Value & \% of Total \\
\hline Genome size (bp) & $8,771,651$ & $100.00 \%$ \\
DNA coding region (bp) & $7,756,096$ & $88.42 \%$ \\
DNA G+C content (bp) & $4,131,717$ & $47.10 \%$ \\
Number of replicons & 4 & \\
Extrachromosomal elements & 3 & \\
Total genes & 6,918 & $100.00 \%$ \\
RNA genes & 106 & $0.87 \%$ \\
rRNA operons & 2 & \\
Protein-coding genes & 6,858 & $99.13 \%$ \\
Pseudo genes & 106 & $1.53 \%$ \\
Genes with function prediction & 4,054 & $58.60 \%$ \\
Genes in paralog clusters & 325 & $4.70 \%$ \\
Genes assigned to COGs & 3,905 & $56.45 \%$ \\
Genes assigned Pfam domains & 4,456 & $64.41 \%$ \\
Genes with signal peptides & 2,889 & $41.76 \%$ \\
Genes with transmembrane helices & 1,588 & $22.95 \%$ \\
CRISPR repeats & 8 & \\
\hline
\end{tabular}

Table 4. Number of genes associated with the general COG functional categories

\begin{tabular}{lrrl}
\hline Code & value & \% age & Description \\
\hline J & 171 & 4.0 & Translation, ribosomal structure and biogenesis \\
A & 0 & 0.0 & RNA processing and modification \\
K & 349 & 8.2 & Transcription \\
L & 190 & 4.4 & Replication, recombination and repair \\
B & 2 & 0.1 & Chromatin structure and dynamics \\
D & 26 & 0.6 & Cell cycle control, cell division, chromosome partitioning \\
Y & 0 & 0.0 & Nuclear structure \\
V & 146 & 3.4 & Defense mechanisms \\
T & 291 & 6.8 & Signal transduction mechanisms \\
M & 333 & 7.8 & Cell wall/membrane/envelope biogenesis \\
N & 20 & 0.5 & Cell motility \\
Z & 0 & 0.0 & Cytoskeleton \\
W & 0 & 0.0 & Extracellular structures \\
U & 64 & 1.5 & Intracellular trafficking, secretion, and vesicular transport \\
O & 161 & 3.8 & Posttranslational modification, protein turnover, chaperones \\
C & 216 & 5.1 & Energy production and conversion \\
G & 271 & 6.3 & Carbohydrate transport and metabolism \\
E & 306 & 7.2 & Amino acid transport and metabolism \\
F & 79 & 1.9 & Nucleotide transport and metabolism \\
H & 150 & 3.5 & Coenzyme transport and metabolism \\
I & 133 & 3.1 & Lipid transport and metabolism \\
P & 254 & 5.9 & Inorganic ion transport and metabolism \\
Q & 95 & 2.2 & Secondary metabolites biosynthesis, transport and catabolism \\
R & 596 & 13.9 & General function prediction only \\
S & 423 & 9.9 & Function unknown \\
- & 3,013 & 43.6 & Not in COGs \\
\hline
\end{tabular}




\section{Acknowledgements}

We would like to gratefully acknowledge the help of Anja Frühling (DSMZ) for growing H. hydrossis cultures. This work was performed under the auspices of the US Department of Energy Office of Science, Biological and Environmental Research Program, and by the University of California, Lawrence Berkeley National Laboratory under contract No. DE-AC02-05CH11231, Lawrence

\section{References}

1. van Veen WL, van der Kooij D, Geuze ECWA, van der Vlies AW. Investigations on the sheathed bacterium Haliscomenobacter hydrossis. Antonie van Leeuwenhoek 1973; 39:207-216. PubMed

2. Skerman VBD, McGowan V, Sneath PHA, eds. Approved Lists of Bacterial Names. Int J Syst Bacteriol 1980; 30:225-420. doi:10.1099/00207713$\underline{30-1-225}$

3. Emmrich $M$, Ziegler M, Rüden H. Filamentous bacteria in activated sludge (bulking sludge). I. Occurrence of filamentous bacteria in a sewage treatment plant working with the activated sludge process (plug flow system). Zentralbl. Bakteriol Microbiol Hyg B 1983; 177:436-450.

4. Fourest E, Craperi D, Deschamps-Roupert C, Pisicchio JL, Lenon G. Occurrence and control of filamentous bulking in aerated wastewater treatment plants of the French paper industry. Water Sci Technol 2004; 50:29-37. PubMed

5. Kotay SM, Datta T, Choi J, Goel R. Biocontrol of biomass bulking caused by Haliscomenobacter hydrossis using a newly isolated lytic bacteriophage. Water Res 2011; 45:694-704. PubMed doi:10.1016/j.watres.2010.08.038

6. Altschul SF, Gish W, Miller W, Myers EW, Lipman DJ. Bascic local alignment search tool. J Mol Biol 1990; 215:403-410. PubMed

7. DeSantis TZ, Hugenholtz $P$, Larsen $N$, Rojas $M$, Brodie EL, Keller K, Huber T, Dalevi D, Hu P, Andersen GL. Greengenes, a chimera-checked $16 \mathrm{~S}$ rRNA gene database and workbench compatible with ARB. Appl Environ Microbiol 2006; 72:5069-5072. PubMed doi:10.1128/AEM.03006-05

8. Porter MF. An algorithm for suffix stripping. Program: electronic library and information systems 1980; 14:130-137.

9. Castresana J. Selection of conserved blocks from multiple alignments for their use in phylogenetic analysis. Mol Biol Evol 2000; 17:540-552. PubMed
Livermore National Laboratory under Contract No. DEAC52-07NA27344, and Los Alamos National Laboratory under contract No. DE-AC02-06NA25396, UTBattelle and Oak Ridge National Laboratory under contract DE-AC05-000R22725, as well as German Research Foundation (DFG) INST 599/1-1.

10. Lee C, Grasso C, Sharlow MF. Multiple sequence alignment using partial order graphs. Bioinformatics 2002; 18:452-464. PubMed doi:10.1093/bioinformatics/18.3.452

11. Stamatakis A, Hoover P, Rougemont J. A rapid bootstrap algorithm for the RAxML Web servers. Syst Biol 2008; 57:758-771. PubMed doi:10.1080/10635150802429642

12. Hess PN, De Moraes Russo CA. An empirical test of the midpoint rooting method. Biol / Linn SoC Lond 2007; 92:669-674. doi:10.1111/j.10958312.2007.00864.x

13. Pattengale ND, Alipour M, Bininda-Emonds ORP, Moret BME, Stamatakis A. How many bootstrap replicates are necessary? Lect Notes Comput Sci 2009; 5541:184-200. doi:10.1007/978-3-642$\underline{02008-7 \_13}$

14. Swofford DL. PAUP*: Phylogenetic Analysis Using Parsimony (*and Other Methods), Version 4.0 b10. Sinauer Associates, Sunderland, 2002.

15. Liolios K, Chen IM, Mavromatis K, Tavernarakis $\mathrm{N}$, Hugenholtz P, Markowitz VM, Kyrpides NC. The Genomes On Line Database (GOLD) in 2009: status of genomic and metagenomic projects and their associated metadata. Nucleic Acids Res 2010; 38:D346-D354. PubMed doi:10.1093/nar/gkp848

16. Field D, Garrity G, Gray T, Morrison N, Selengut J, Sterk P, Tatusova T, Thomson N, Allen MJ, Angiuoli SV, et al. The minimum information about a genome sequence (MIGS) specification. Nat Biotechnol 2008; 26:541-547. PubMed doi:10.1038/nbt1360

17. Garrity G. NamesforLife. BrowserTool takes expertise out of the database and puts it right in the browser. Microbiol Today 2010; 37:9.

18. Woese CR, Kandler O, Wheelis ML. Towards a natural system of organisms: proposal for the domains Archaea, Bacteria, and Eucarya. Proc Natl Acad Sci USA 1990; 87:4576-4579. PubMed doi:10.1073/pnas.87.12.4576 
19. Garrity GM, Holt JG. The Road Map to the Manual. In: Garrity GM, Boone DR, Castenholz RW (eds), Bergey's Manual of Systematic Bacteriology, Second Edition, Volume 1, Springer, New York, 2001, p. 119-169.

20. Garrity GM, Holt JG. Taxonomic Outline of the Archaea and Bacteria. In: Garrity GM, Boone DR, Castenholz RW (eds), Bergey's Manual of Systematic Bacteriology, Second Edition, Volume 1, Springer, New York, 2001, p. 155-166.

21. Euzéby JP. List of Bacterial Names with Standing in Nomenclature: a folder available on the Internet. Int J Syst Bacteriol 1997; 47:590-592. PubMed doi:10.1099/00207713-47-2-590

22. BAuA. 2010. Classification of bacteria and archaea in risk groups. TRBA 466.

23. Ashburner M, Ball CA, Blake JA, Botstein D, Butler $\mathrm{H}$, Cherry JM, Davis AP, Dolinski K, Dwight SS, Eppig JT, et al. Gene Ontology: tool for the unification of biology. Nat Genet 2000; 25:25-29. $\underline{\text { PubMed doi:10.1038/75556 }}$

24. Kämpfer P. Physiological and chemotaxonomic characteriation of filamentous bacteria belonging to the genus Hyalscomenobacter. Syst Appl Microbiol 1995; 18:363-367.

25. Klenk HP, Göker M. En route to a genome-based classification of Archaea and Bacteria? Syst Appl Microbiol 2010; 33:175-182. PubMed doi:10.1016/j.syapm.2010.03.003

26. Wu D, Hugenholtz P, Mavromatis K, Pukall R, Dalin E, Ivanova NN, Kunin V, Goodwin L, Wu $M$, Tindall BJ, et al. A phylogeny-driven genomic encyclopaedia of Bacteria and Archaea. Nature 2009; 462:1056-1060. PubMed doi:10.1038/nature08656

27. List of growth media used at DSMZ: http://www.dsmz.de/microorganisms/media_list.p hp.
28. Gemeinholzer B, Dröge G, Zetzsche H, Haszprunar G, Klenk HP, Güntsch A, Berendsohn WG, Wägele JW. The DNA Bank Network: the start from a German initiative. Biopreservation and Biobanking 2011; 9:51-55.

doi:10.1089/bio.2010.0029

29. JGI website. http://www.jgi.doe.gov.

30. The Phred/Phrap/Consed software package. http://www.phrap.com

31. Zerbino DR, Birney E. Velvet: algorithms for de novo short read assembly using de Bruijn graphs. Genome Res 2008; 18:821-829. PubMed doi:10.1101/gr.074492.107

32. Han C, Chain P. Finishing repeat regions automatically with Dupfinisher. In: Proceeding of the 2006 international conference on bioinformatics \& computational biology. Arabnia HR, Valafar $\mathrm{H}$ (eds), CSREA Press. June 26-29, 2006: 141-146.

33. Lapidus A, LaButti K, Foster B, Lowry S, Trong S, Goltsman E. POLISHER: An effective tool for using ultra short reads in microbial genome assembly and finishing. AGBT, Marco Island, FL, 2008.

34. Hyatt D, Chen GL, LoCascio PF, Land ML, Larimer FW, Hauser LJ. Prodigal: prokaryotic gene recognition and translation initiation site identification. BMC Bioinformatics 2010; 11:119. PubMed doi:10.1186/1471-2105-11-119

35. Pati A, Ivanova NN, Mikhailova N, Ovchinnikova G, Hooper SD, Lykidis A, Kyrpides NC. GenePRIMP: a gene prediction improvement pipeline for prokaryotic genomes. Nat Methods 2010; 7:455-457. PubMed doi:10.1038/nmeth.1457

36. Markowitz VM, Ivanova NN, Chen IMA, Chu K, Kyrpides NC. IMG ER: a system for microbial genome annotation expert review and curation. Bioinformatics 2009; 25:2271-2278. PubMed doi:10.1093/bioinformatics/btp393 